\title{
Adiponectin alleviated renal cell apoptosis and inflammation via inactivation of JAK2/STAT3 signal pathway in an acute pyelonephritis mouse model
}

\author{
Guoyu Dai, Yao He \\ Department of Urology, Xiangya Hospital, Central South University, Changsha, China \\ Contributions: (I) Conception and design: Both authors; (II) Administrative support: Y He; (III) Provision of study materials: G Dai; (IV) Collection \\ and assembly of data: G Dai; (V) Data analysis and interpretation: Y He; (VI) Manuscript writing: Both authors; (VII) Final approval of manuscript: \\ Both authors. \\ Correspondence to: Yao He. Department of Urology, Xiangya Hospital, Central South University, Changsha 410008, China. Email: heyao1984@163.com.
}

\begin{abstract}
Background: Acute pyelonephritis (APN), an acute and severe kidney infection, is usually treated with antibiotics. However, APN treatment has become increasingly challenging because of bacterial resistance. Adiponectin, an adipokine, has recently been reported to exhibit profound anti-inflammatory and antiapoptotic effects. However, the effect of adiponectin on the outcomes of APN treatment remains unclear. In this study, we aimed to investigate the effects of adiponectin on APN and the mechanisms underlying these effects.
\end{abstract}

Methods: Wild-type C57 mice and adiponectin-knockout (KO) mice were divided into 6 groups: the wildtype control group, the wild-type model group, the wild-type adiponectin intervention group, the KO control group, the KO model group, and the adiponectin-KO intervention group. We measured white blood cell (WBC) and neutrophil counts (NC) using a multispecies hematology analyzer; tumor necrosis factor- $\alpha$ (TNF- $\alpha$ ) and interleukin-6 (IL-6) using enzyme-linked immunosorbent assay (ELISA); blood urea nitrogen (BUN) and serum creatinine (SCr) levels using colorimetry; and the protein levels of JAK2, STAT3, p-JAK2, p-STAT3, Bcl-2, and Bax in renal tissues using western blot analysis. Apoptotic cells were detected using the transferase-mediated dUTP nick end labelling (TUNEL) assay.

Results: Compared to the wild-type mice, the KO mice showed a more severe inflammatory response and kidney damage after Escherichia coli infection. After treatment with exogenous adiponectin injection, the inflammatory response, oxidative stress, and kidney damage were partly alleviated. Adiponectin KO led to JAK2/STAT3 signaling activation, and exogenous adiponectin administration inactivated JAK2/STAT3 signaling in the APN model. APN can lead to an increase in the level of the protein Bax and a decrease in the level of the bcl-2 protein, thereby increasing apoptosis; this effect was inhibited by adiponectin.

Conclusions: Through use of a pyelonephritis mouse model, we demonstrated that adiponectin might alleviate renal cell apoptosis and inflammatory response by inactivating the JAK2/STAT3 signaling pathway.

Keywords: Acute pyelonephritis (APN); JAK2/STAT3 signaling; adiponectin; apoptosis; inflammatory response

Submitted Jun 04, 2021. Accepted for publication Aug 05, 2021.

doi: $10.21037 /$ atm-21-3325

View this article at: https://dx.doi.org/10.21037/atm-21-3325

\section{Introduction}

Urinary tract infections (UTIs) are the most common community-and hospital-acquired bacterial infections (1), and when the infection occurs in the upper urinary tract it causes acute pyelonephritis (APN), a life-threatening complication (2). The most common cause of APN is Escherichia coli infection (3). Typical clinical manifestations suggestive of pyelonephritis are fever (temperature $>38^{\circ} \mathrm{C}$ ), 
chills, flank pain, and nausea or vomiting (4). Furthermore, APN often causes serious conditions, including urosepsis (5). Current therapy for APN is based on antibiotics, However, owing to the increase in bacterial resistance to antibiotics, APN treatment is facing growing challenges (6). Therefore, it is important to develop a novel treatment for APN.

Numerous studies have shown that obesity increases the risk and degree of renal injury in patients with $\operatorname{APN}(7,8)$. Recent studies have indicated that a lower adiponectin $(\mathrm{ADPN})$ level in obese patients is associated with their higher risk of APN (9). Adiponectin accounts for $0.01 \%$ of the total plasma proteins in humans; it is a hormone that is primarily secreted by adipose tissue (10-12). In clinical practice, the screening for ADPN deficiency normally is not required. A growing body of evidence suggests that ADPN suppresses cell death, inhibits inflammation, promotes cell survival, and protects cells against pathological events $(13,14)$. However, the role of ADPN in the pathogenesis of APN has not barely investigated.

The JAK2/STAT3 signaling pathway has been reported to regulate several biological processes, including cell survival, cell proliferation, and the cell cycle (15). In addition, it plays critical roles in apoptosis and inflammation (16). Recently, it was suggested that ADPN reduces apoptosis and inhibits inflammation via the JAK2/STAT3 signal pathway in hippocampal HT22 cells (17). Therefore, we hypothesized that ADPN might affect APN by reducing apoptosis and inflammation mediated by the JAK2/STAT3 signaling pathway.

In this study, we demonstrated that ADPN exhibited a renal protection and therapeutic potential for APN in the mouse model, which is commonly established by directly instilling E. coli into the bladder. The mechanisms by which ADPN protects the kidney from E. coli-induced injury may be due to its capability of reducing renal cell apoptosis and inflammatory responses mediated by the JAK2/STAT3 signaling pathway. We present the following article in accordance with the ARRIVE reporting checklist (available at https://dx.doi.org/10.21037/atm-21-3325).

\section{Methods}

\section{Primary reagent}

Adiponectin was purchased from Sigma-Aldrich (St. Louis, MO, USA). E. coli (ATCC25922) was purchased from Shanghai Beinuo Biochemical Co., Ltd. (Shanghai, China).

\section{Animal preparation and experimental protocol}

Female C57 [wild type (WT)] mice (24 \pm 4.3 g, 6-8 weeks old) were obtained from the Department of Laboratory Animal Unit of the Central South University, China. Female ADPNknockout (KO) mice (KOCMP-11450-Adipoq-B6N-VA, $26 \pm 3.7 \mathrm{~g}, 6-8$ weeks old) were purchased from Cyagen Biosciences Inc. (Guangzhou China). The Ethics Committee of Experimental Animal Management and Animal Welfare of Central South University reviewed and approved the study (NO: 201703526). All animal experiments were carried out in accordance with the National Institutes of Health's Guidelines for the Care and Use of Laboratory Animals. A protocol was prepared before the study without registration. All mice were housed in a standard environment at $25^{\circ} \mathrm{C}$ and humidity of $50 \%$, in a 12 -h light/dark cycle with free access to water and food. The experiment operation has been described (18). Briefly, after anesthesia, WT mice or $\mathrm{KO}$ mice were infected with an intravesical inoculation of $0.05 \mathrm{~mL}$ of a freshly prepared solution containing $1 \times 10^{6}$ colony-forming unit (CFU)/mL of E. coli (ATCC 25922) or $0.05 \mathrm{~mL}$ of sterile phosphate-buffered saline (PBS) through a soft polyethylene catheter. The catheter was withdrawn, and the mice were allowed feed and water ad libitum. Any mouse that was not successfully infected by E. coli using this procedure or died from 0 to $72 \mathrm{~h}$ after bacterial or PBS inoculation was excluded from statistical analysis. Ten animals per group were included for data analysis based on the sample size calculation as described in literature (19). The success of bacterial infection such as the sign of fever was assessed in a blinded manner by GD. In the WT control group, the WT model group, the $\mathrm{KO}$ control group, and the $\mathrm{KO}$ model group mice were subcutaneously injected with $10.0 \mathrm{mg} / \mathrm{kg}$ of sterile PBS every day for 3 consecutive days. In the KO ADPN intervention group and the WT ADPN intervention group, mice were injected subcutaneously with $10.0 \mathrm{mg} / \mathrm{kg}$ of ADPN every day for 3 consecutive days after intravesical bacteria injection. All mice were sacrificed on the third day after intravesical inoculation with bacteria or administration of PBS. Blood samples and renal tissues were collected for use.

\section{Visceral index}

The kidney was weighed, and the visceral index of the kidney was calculated using the following formula: viscera index $=$ viscera weight $(\mathrm{g}) /$ mice weight $(\mathrm{g}) \times 100 \%$. 


\section{Hematological analysis}

Blood was taken from the ophthalmic artery. The levels of white blood cells (WBC) and neutrophil counts (NC) in the blood were measured using a multispecies hematology analyzer (Sysmex XS-500i, Japan).

\section{Assessment of renal injury indicators}

Renal injury was indicated by the levels of blood urea nitrogen (BUN) and serum creatinine ( $\mathrm{SCr}$ ), The serum levels of BUN and SCr were tested using colorimetry assays (AU5800, Beckman Coulter, Brea, CA, USA).

\section{Serum cytokine detection}

Interleukin-6 (IL-6) and tumor necrosis factor- $\alpha$ (TNF- $\alpha$ ) concentrations in serum were measured using an enzymelinked immunosorbent assay (ELISA) kit (Abcam, Cambridge, UK) according to the manufacturer's protocol. Absorbance was measured using an ELISA plate reader at an optical density (OD) $650 \mathrm{~nm}$.

\section{Renal pathology score}

Kidney tissues were stained with hematoxylin and eosin (HE) for histopathological observation. Briefly, kidney tissues were fixed in a $4 \%$ paraformaldehyde solution and then embedded in paraffin. 4- $\mu \mathrm{m}$ sections were stained in a hematoxylin solution for 4 minutes, and counterstained with eosin for 3 minutes. Inflammatory changes in each kidney were summed from semiquantitative scoring of 10 randomly selected fields in a blinded manner by $\mathrm{YH}$. The following scoring system was used: 0 , normal; 1 , inflammation, indicated by leukocyte infiltrates, in any pelvic region; 2, parenchymal inflammation adjacent to the fornices; and 3, inflammation in cortex and medulla (6).

\section{Transferase-mediated dUTP nick end labelling (TUNEL) assay}

TUNEL staining was conducted by with a TUNEL Kit (Yisheng Technology Co., Ltd., Shanghai, China). Briefly, sections were permeabilized with $0.1 \%$ TritonX-100 for 2 minutes. TUNEL reaction mixture was added to the sections and incubated in a chamber at $37^{\circ} \mathrm{C}$ for 1 hour. Then the coverslips were mounted onto the glass slides. The sections were kept away from light during the experiment. The apoptotic cells were indicated by strong and dense fluorescence staining as compared with background fluorescence. The number of apoptotic cells was counted using a fluorescence microscope (DX51, Olympus, Tokyo, Japan).

\section{Western blot assay}

Kidney tissues were lysed in lysis buffer (Beyotime, China). Proteins $(20 \mu \mathrm{g})$ were separated by $10 \%$ SDSPAGE and transferred to a PVDF membrane. After blocking, the membranes were incubated overnight at $4{ }^{\circ} \mathrm{C}$ with primary antibodies against Bcl-2 (1:500, Abcam), Bax (1:500, Abcam), JAK2 (1:1,000, Abcam), p-JAK2 (1:500, Abcam), STAT3 (1:1,000, Abcam), p-STAT3 (1:500, Abcam), and anti- $\beta$ actin (1:3,000, Abcam). Subsequently, the membranes were incubated for 1 hour with horseradish peroxidase (HRP) conjugated secondary antibodies [1:5,000 goat anti-rabbit immunoglobin $\mathrm{G}$ ( $\mathrm{IgG}$ ), or 1:3,000 goat anti-mouse IgG; Millipore]. Protein bands were detected using enhanced chemiluminescent reagents (Millipore). Each target protein was identified based on their molecular weight in the blotting.

\section{Statistical analysis}

All results were expressed as mean \pm standard deviation (SD). Comparisons among different groups were made using oneway analysis of variance (ANOVA) followed by the StudentNewman-Keuls test, or using the nonparametric rank sum test as appropriate. $\mathrm{P}$ value $<0.05$ was considered to be significant. All analyses were performed using GraphPad Prism 6.0.

\section{Results}

\section{ADPN reduced E. coli-induced renal injury in the APN model}

Compared to the PBS injection, E. coli injection for inducing APN resulted in a significantly higher visceral index in mice $(\mathrm{P}<0.05$; Figure $1 A)$, indicating that the APN model was successfully established. As shown in Figure $1 A$, the visceral index in the $\mathrm{KO}$ model group was higher than that in the WT model group $(\mathrm{P}<0.05$; Figure $1 A)$. In addition, the visceral index was reduced after $\mathrm{ADPN}$ intervention $(\mathrm{P}<0.05)$. To further explore the protective effect of ADPN, $\mathrm{SCr}$ and BUN were detected in the blood. The results suggested that $\mathrm{ADPN} \mathrm{KO}$ had no influence on the levels of SCr and BUN under normal conditions. The levels of 

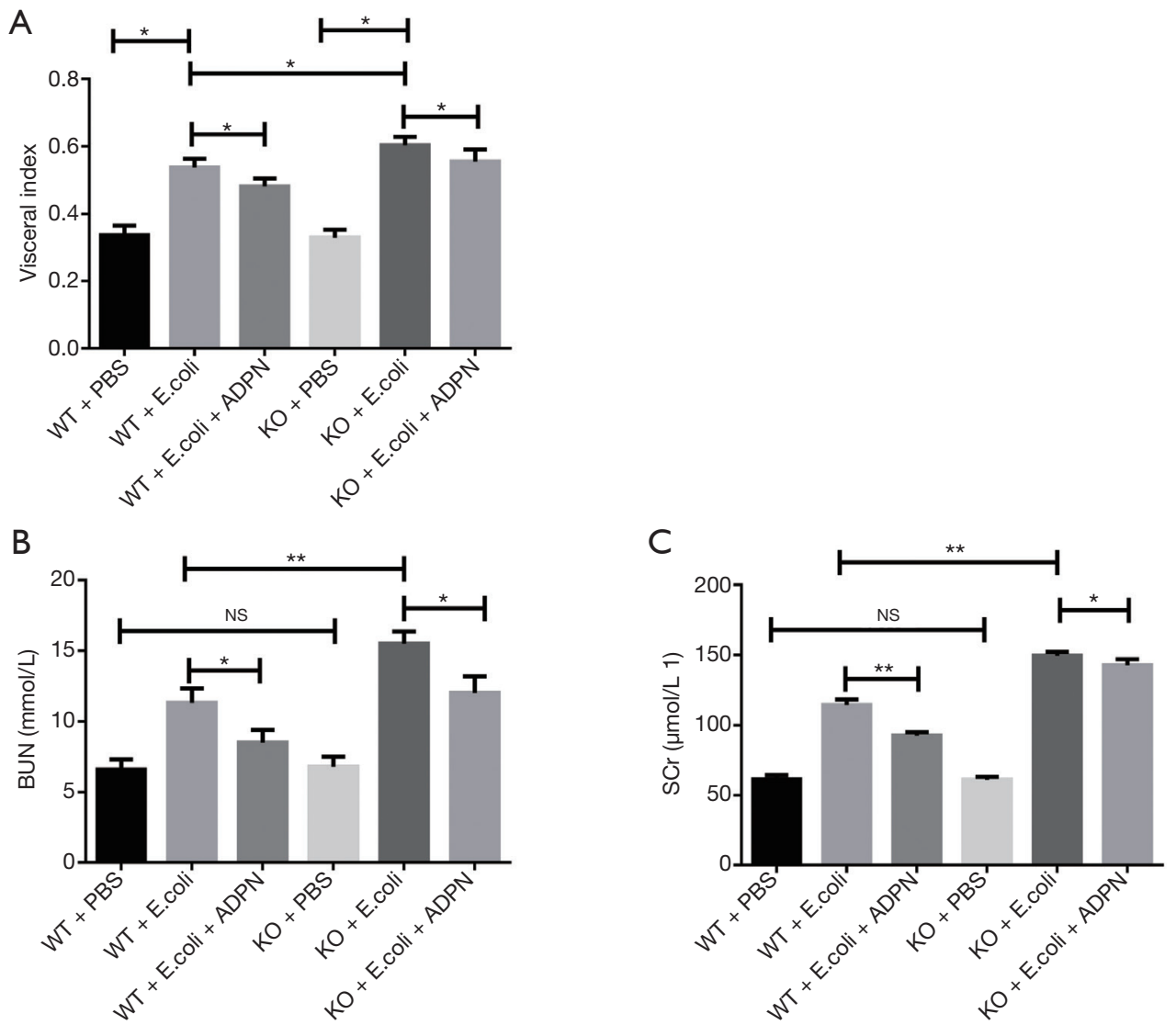

Figure 1 The effect of ADPN on kidney function and damage. The effect of ADPN on visceral index (A), and the level of BUN (B) and $\mathrm{SCr}(\mathrm{C})$ in blood were tested using colorimetry assays. The data are presented as mean $\pm \mathrm{SD}(\mathrm{n}=10)$. ${ }^{*} \mathrm{P}<0.05,{ }^{* *} \mathrm{P}<0.01, \mathrm{NS}$ represents no significant difference. ADPN, adiponectin; WT, wild type; PBS, phosphate-buffered saline; KO, knockout; BUN, blood urea nitrogen; SCr, serum creatinine; SD, standard deviation.

BUN and SCr in the KO and WT groups were significantly increased after APN treatment $(\mathrm{P}<0.05$; Figure 1B,1C). After treatment with ADPN, the levels of BUN and SCr were significantly decreased $(\mathrm{P}<0.05$; Figure $1 B, 1 C)$.

\section{ADPN reduced E. coli-induced renal pathological injury score in the APN model}

Furthermore, the renal pathological status was examined. As shown in Figure $2 A$ and $2 B$, no inflammatory cells were observed in the kidney tissues of the WT control group and the KO control group. The tissues presented clear structures without degeneration, atrophy, or swelling. In the WT model group, the inflammatory cells were primarily concentrated in the renal medulla and the junction between the renal cortex and renal medulla, with fewer than 2 abscesses at a magnification of 200x. In contrast, the KO model group exhibited severe and extensive tissue damage, glomerular hemorrhage, presence of polymorphonuclear leukocytes, interstitial inflammation, and glomerular necrosis; the inflammatory cells penetrated the renal medulla and the renal cortex, with larger abscesses being present. After treatment with ADPN, the signs of kidney damage were alleviated. Subsequently, the kidney injury scores in the different groups were calculated, as presented in Figure $2 C$.

\section{ADPN reduced E. coli-induced inflammation response in the APN model}

Treatment with $E$. coli resulted in an increase in WBC count and $\mathrm{NC}$ in the WT and $\mathrm{KO}$ groups $(\mathrm{P}<0.05$; Figure $3 A, 3 B)$. Without treatment with $E$. coli, ADPN KO exerted no influence on the $\mathrm{WBC}$ count and $\mathrm{NC}(\mathrm{P}>0.05$; Figure $3 A, 3 B)$. However, the ADPN KO led to a significant increase in 
A
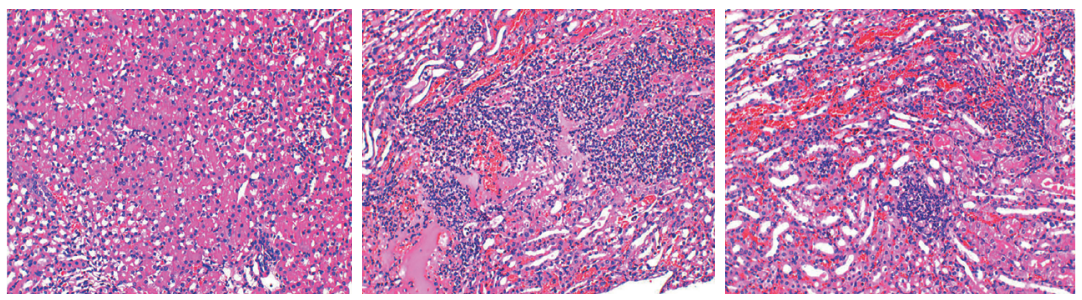

$\times 200$
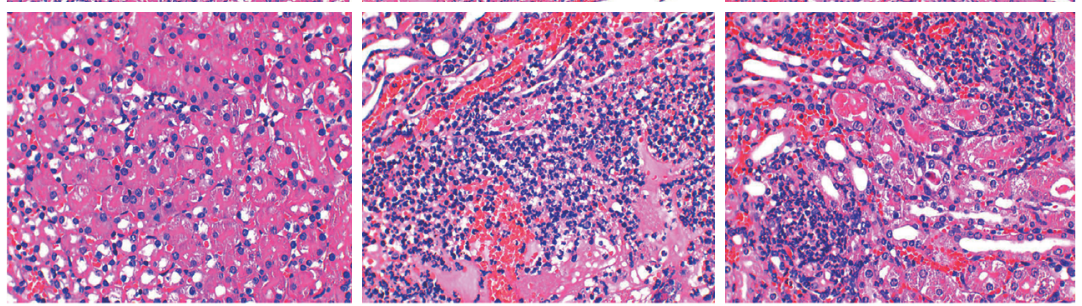

$\mathrm{KO}+\mathrm{PBS}$

$\mathrm{KO}+$ E.coli

$\mathrm{KO}+\mathrm{E}$. coli $+\mathrm{ADPN}$

B
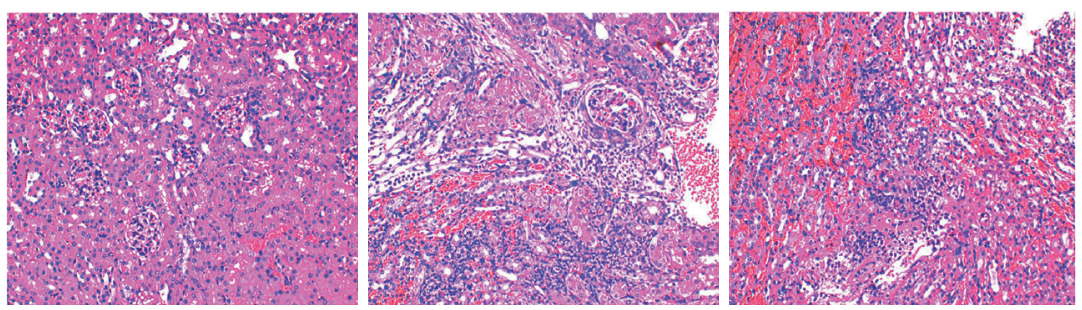

$\times 400$
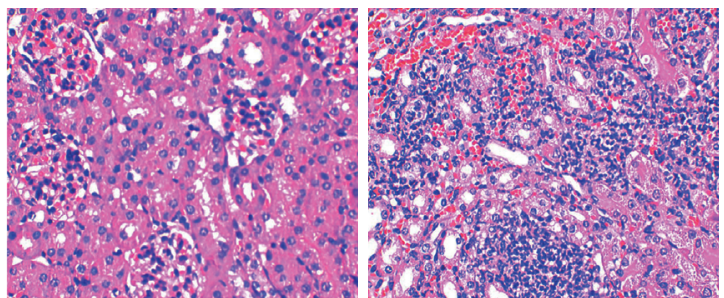

WT + PBS

WT + E.coli

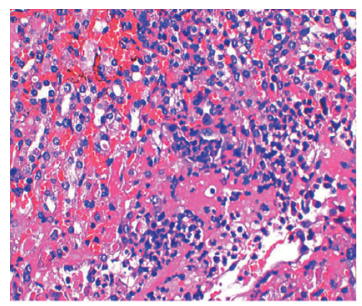

$\times 200$

C

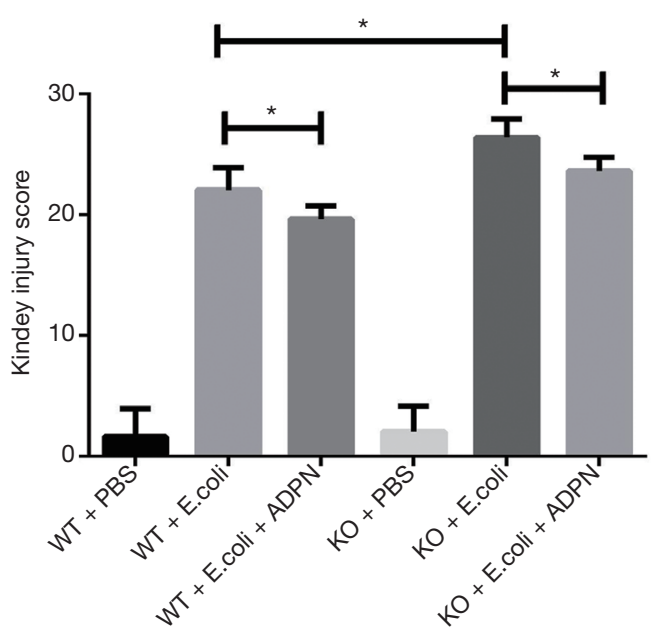

WT + E.coli + ADPN

Figure 2 The effect of ADPN on renal pathological status and kidney injure score. The kidney gross histopathology of KO mice (A) and the kidney gross histopathology of WT mice (B). Representative kidney sections were stained with HE ( $\times 200$ magnification \& $\times 400$ magnification). The changes of kidney injury score (C). The data are presented as mean \pm SD $(n=10)$. ${ }^{*} \mathrm{P}<0.05$. ADPN, adiponectin; KO, knockout; WT, wild type; SD, standard deviation. 


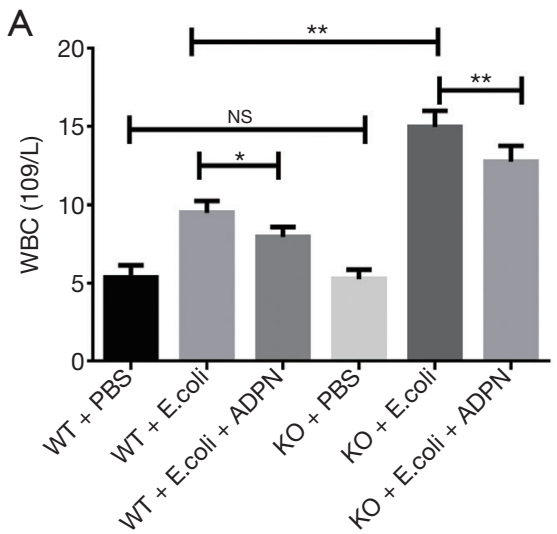

B

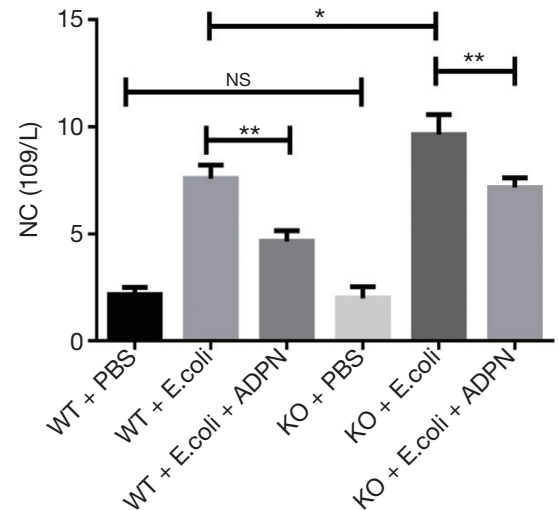

C
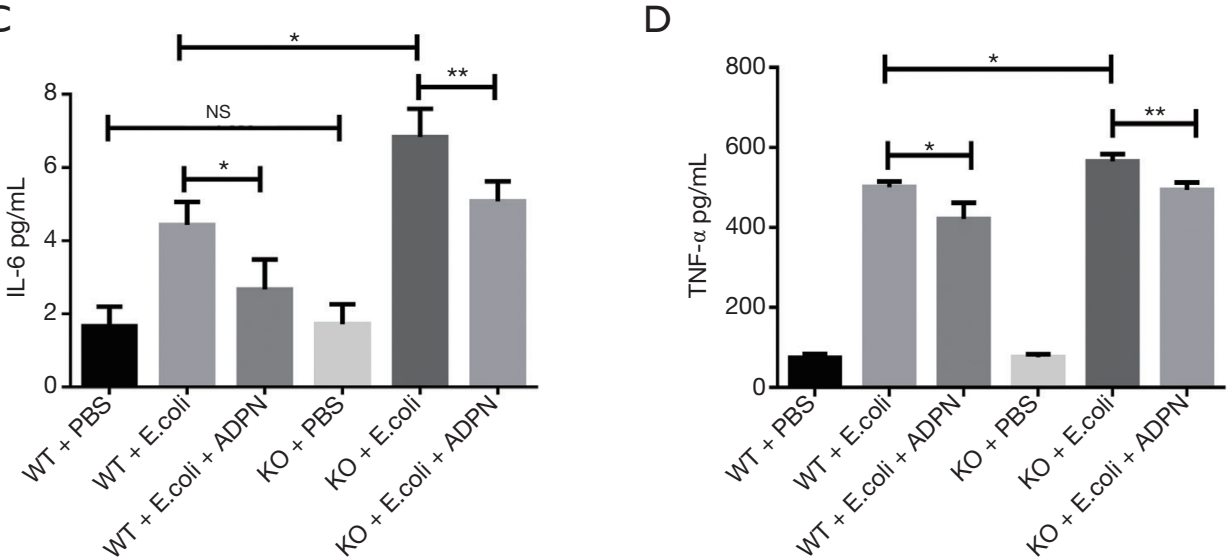

Figure 3 The effect of ADPN on inflammatory response. WBC (A) and NC (B) count as measured by multispecies hematology analyzer. The concentrations of IL-6 (C) and TNF- $\alpha$ (D) were detected by ELISA. The data are presented as mean \pm SD $(n=10)$. ${ }^{*} \mathrm{P}<0.05$; ${ }^{* *} \mathrm{P}<0.01$, NS represents no significant difference. ADPN, adiponectin; WT, wild type; PBS, phosphate-buffered saline; KO, knockout; WBC, white blood cell; NC, neutrophil counts; IL-6, interleukin-6; TNF- $\alpha$, tumor necrosis factor- $\alpha$; ELISA, enzyme-linked immunosorbent assay; SD, standard deviation.

the WBC count and NC in the APN model $(\mathrm{P}<0.05)$. Administration of ADPN significantly decreased the WBC count and NC induced by E. coli. Subsequently, the IL-6 and TNF- $\alpha$ levels in the plasma were measured using ELISA. In the APN model group, ADPN KO promoted the secretion of IL- 6 and TNF- $\alpha$, and treatment with ADPN partly reduced the secretion of IL- 6 and TNF- $\alpha$ induced by $E$. coli. All the results indicated that ADPN reduced the E. coliinduced inflammation response by decreasing the secretion of IL-6 and TNF- $\alpha$ in the APN model $(\mathrm{P}<0.05$; Figure $3 C, 3 D)$.

\section{$A D P N$ reduced the Bax to Bcl-2 ratio and decreased $E$. coli-induced renal cell apoptosis}

The apoptosis of cells in the kidney tissues was detected.
After treatment with $E$. coli, the KO group mice showed more apoptotic cells in the kidney tissues compared with the WT model group. However, ADPN administration decreased the apoptosis rate (Figure $4 A, 4 B$ ). Furthermore, E. coli significantly increased the ratio of Bax to Bcl-2 in the renal tissue. However, ADPN administration significantly decreased the ratio of Bax to Bcl-2 (Figure 4C,4D).

\section{ADPN reduced E. coli-induced apoptosis of renal cells by inactivating $7 A K 2 / S T A T 3$ signal patbway in the APN model}

To explore the potential mechanisms through which ADPN reduced both apoptosis and inflammation response that were induced by E. coli, JAK2/STAT3 signaling was 
A
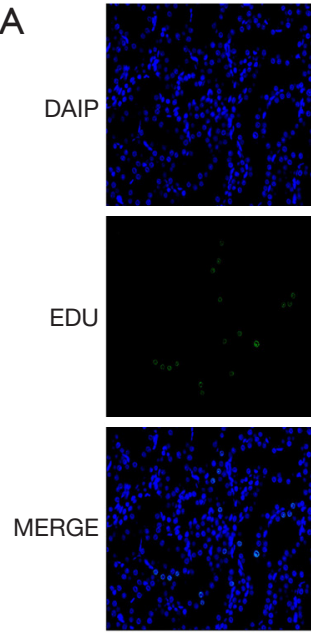

$\mathrm{KO}+\mathrm{PBS}$
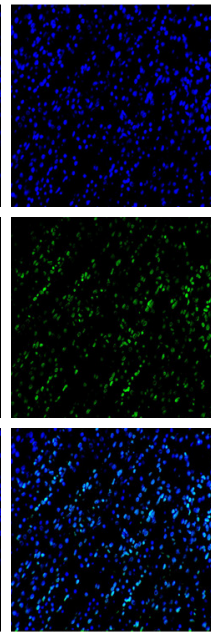

$\mathrm{KO}+\mathrm{E} . \mathrm{coli}$
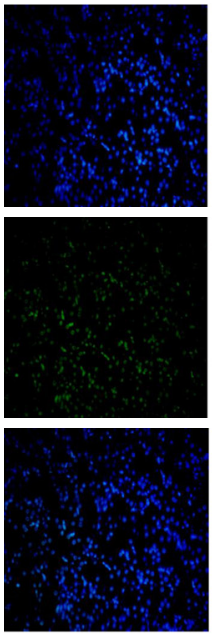

$\mathrm{KO}+\mathrm{E} \cdot \mathrm{coli}+\mathrm{ADPN}$
B
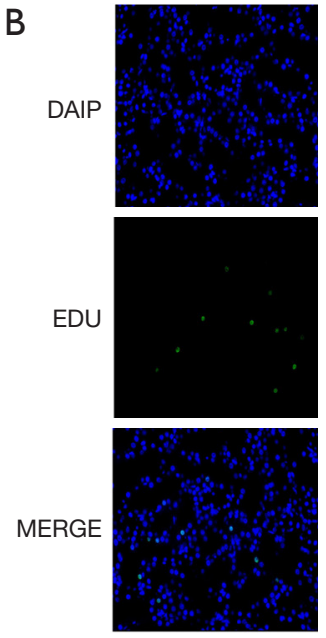

$W T+P B S$
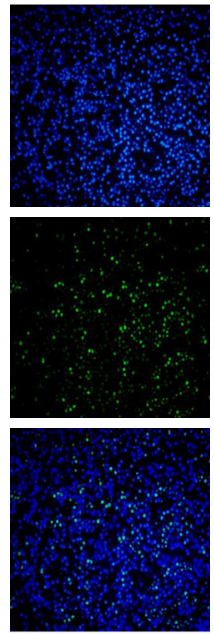

WT + E.coli $\quad W T+$ E.coli + ADPN

C

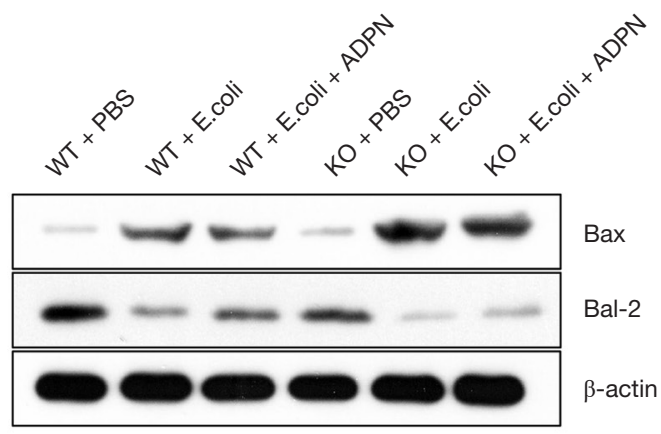

D

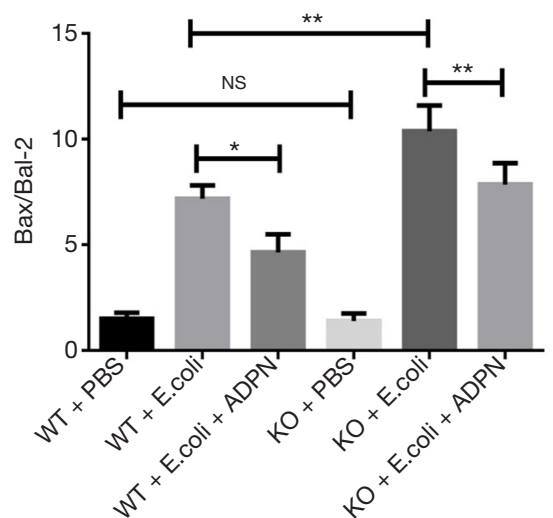

Figure 4 The influence of ADPN on apoptosis in kidney tissues in mice group. The kidney TUNEL staining of KO mice (A) ( $\times 100$ magnification). The kidney TUNEL staining of WT mice (B) ( $\times 100$ magnification). The protein levels of Bax and Bcl2 in kidneys were assessed using western blotting (C). Description of statistical results of western blotting (D). The data are presented as mean \pm SD $(\mathrm{n}=10) .{ }^{*} \mathrm{P}<0.05 ;{ }^{* *} \mathrm{P}<0.01$, NS represents no significant difference. ADPN, adiponectin; WT, wild type; PBS, phosphate-buffered saline; KO, knockout; TUNEL, transferase-mediated dUTP nick end labelling; SD, standard deviation.

evaluated. The results demonstrated that bladder injection with E. coli clearly increased the protein expression of p-JAK2 and p-STAT3 (Figure 5, P<0.05). In addition, ADPN KO significantly increased the expression of p-JAK2 and p-STAT3 in the APN model $(\mathrm{P}<0.05$; Figure 5). Compared with the levels in the WT and KO model groups, the levels of p-JAK2 and p-STAT3 in the WT ADPN intervention group and the ADPN-KO intervention group were markedly decreased $(\mathrm{P}<0.05$; Figure 5).

\section{Discussion}

In our study, a mouse model of APN was successfully created. First, we measured the viscera index, kidney function, and renal pathological status. The results demonstrated that $E$. coli increased the visceral index and damaged kidney function. Adiponectin treatment protected kidney function and rescued the $E$. coli-induced kidney damage. Previous studies have suggested that inflammation plays a major role in the development of tissue damage in E. coli-induced pyelonephritis (20). Consistent with these 

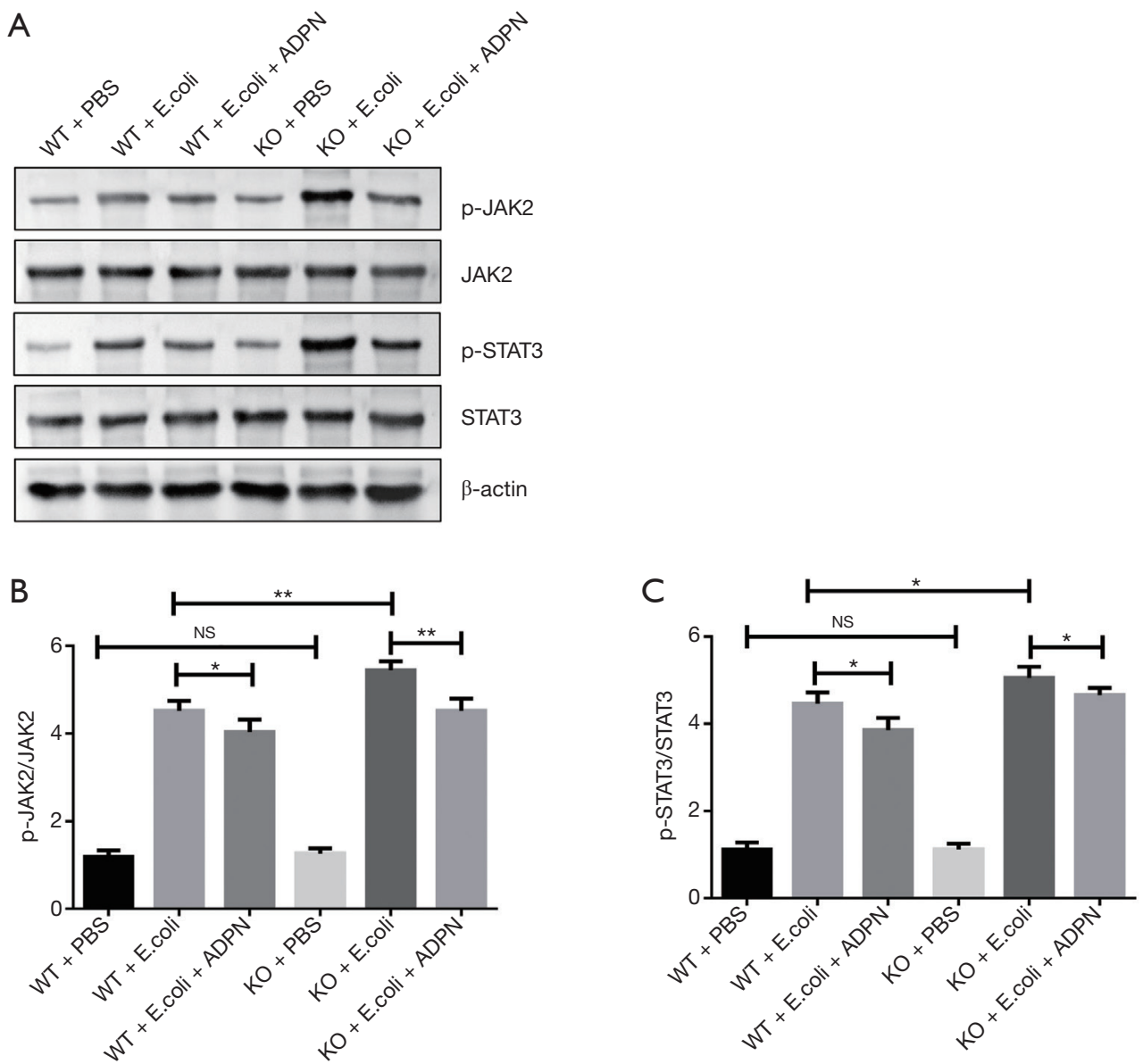

Figure 5 The effect of ADPN on the protein levels of JAK2/STAT3 signaling. The protein level of JAK2, STAT3, p-JAK2, and p-STAT3 were detected by western blotting (A). Description of statistical results of western blotting $(\mathrm{B}, \mathrm{C})$. The data are presented as mean $\pm \mathrm{SD}$, ( $\mathrm{n}=10)$. ${ }^{*} \mathrm{P}<0.05 ;{ }^{* *} \mathrm{P}<0.01$, NS represents no significant difference. ADPN, adiponectin; SD, standard deviation.

studies, our study found that E. coli treatment of the WT and $\mathrm{KO}$ groups resulted in an increase in WBC count and NC. The inflammatory response induced by E. coli was decreased by ADPN, as indicated by the WBC count, NC, and the secretion levels of IL- 6 and TNF- $\alpha$.

Apoptosis, also known as programmed cell death, plays an important role in cell regeneration. Nevertheless, excess apoptosis exerts harmful effects on organ function. Renal cell apoptosis is an important factor affecting kidney function (21). In our study, the apoptosis rate of renal cells was significantly increased in the APN model. Compared with the WT model group, the KO model group exhibited a significantly higher apoptosis level of renal cells. However, ADPN remarkably decreased the apoptosis induced by $E$. coli. In conclusion, these results indicate that ADPN deficiency led to the promotion of inflammation and apoptosis in the APN model. ADPN administration alleviated the inflammation and apoptosis in the APN model, which suggests that ADPN could serve as a potential treatment for APN.

Janus kinases (JAKs) mediate both cytokine and growth factor signalings, which consequently activate the STAT transcriptional factors, resulting in the transcription of several target genes (22). Multiple studies have suggested that the JAK2/STAT3 signaling pathway is related to apoptosis (23-25). The JAK2/STAT3 pathway regulates the levels of the antiapoptotic protein $\mathrm{Bcl}-2$ and the proapoptotic protein Bax, thereby altering the apoptosis of the involved cells (26-29). The JAK2/STAT3 pathway has been reported to be involved in inflammatory infections induced by bacterial infection (30,31). Furthermore, activation of the JAK2/STAT3 signaling pathway can 
increase apoptosis in human renal proximal tubular epithelial cells. Additionally, the antiapoptotic protein Bcl-2 has been reported to be significantly reduced in the renal tubular cells of patients with APN. In addition, a significantly lower Bcl-2 level has been observed in the kidney tissue of pyelonephritic rats compared to that in normal rats (32). To our knowledge, there is currently no drugs targeting JAK2/ STAT3 signaling for ANP treatment. Whereas adiponectin can produce corresponding biological effects through the regulation of the JAK2/STAT3 signaling pathway $(33,34)$. Previous studies have shown that ADPN can antagonize leptin-mediated proliferation through the activation of phosphatase PTP1B, which leads to the inhibition of JAK2 and dephosphorylation of STAT3 (35). Therefore, ADPN might reduce the apoptosis of renal cells by inactivating the JAK2/STAT3 signaling pathway in an APN mouse model. JAK2/STAT3 signaling is additionally related to inflammation. JAK2/STAT3 signaling plays a major role in production of pro-inflammatory cytokines such as IL-1 $\beta$, IL-6, and TNF- $\alpha(36,37)$, and these proinflammatory cytokines induce kidney cell apoptosis. So that the disruption of the JAK2/STAT3 pathways will reduce the pro-inflammatory cytokine production, and the renal cell apoptosis and inflammation will be prevented. IL-6 is immediately and transiently produced following infections and tissue injuries, and it may contribute to host defense through the stimulation of acute phase responses, hematopoiesis, and immune reactions (38). It's reported in previous studies that IL-6 as an important diagnostics biomarker contributes to differentiating between lower UTIs and pyelonephritis (39). In addition, serum IL-6 has shown high sensitivity and specificity in the diagnosis of pyelonephritis $(40,41)$. TNF- $\alpha$ is an inflammatory cytokine produced by macrophages/monocytes during acute inflammation and is an important mediator of the inflammatory response in serious bacterial infections (42). Several studies have shown that TNF- $\alpha$ plays an important role in the diagnosis and treatment of APN (43-46). The results of our study are consistent with those of previous studies; we observed that intravesical inoculation of $E$. coli significantly increased the levels of IL-6 and TNF- $\alpha$ in mice, while after ADPN intervention, the levels of IL-6 and TNF- $\alpha$ significantly decreased. In this study, we observed that the levels of phosphorylated JAK2 and STAT3 in renal tissues were enhanced in APN. Adiponectin suppressed the phosphorylation of JAK2 and STAT3 in renal tissue of APN mice, suggesting that inhibition of the JAK2/ STAT3 pathway may mediate the antiapoptotic and anti- inflammatory properties of ADPN.

\section{Conclusions}

Our study demonstrated that ADPN protects the kidney from damage induced by $E$. coli in an APN animal model. These protective effects can be attributed to the antiinflammatory and anti-apoptotic effects of ADPN. The data shown in this study suggests that ADPN might serve as an attractive candidate for APN treatment. However, further studies are required to assess the effectiveness of ADPN in the treatment of ANP.

\section{Acknowledgments}

The authors thank the laboratory of the Central South University.

Funding: This work was supported by the National Natural Science Foundation of Hunan (no. 2020JJ5895).

\section{Footnote}

Reporting Checklist: The authors have completed the ARRIVE reporting checklist. Available at https://dx.doi. org/10.21037/atm-21-3325

Data Sharing Statement: available at https://dx.doi. org/10.21037/atm-21-3325

Conflicts of Interest: Both authors have completed the ICMJE uniform disclosure form (available at https://dx.doi. org/10.21037/atm-21-3325). The authors report funding support from the National Natural Science Foundation of Hunan (No. 2020JJ5895). The authors have no other conflicts of interest to declare.

Ethical Statement: The authors are accountable for all aspects of the work in ensuring that questions related to the accuracy or integrity of any part of the work are appropriately investigated and resolved. The Ethics Committee of Experimental Animal Management and Animal Welfare of Central South University reviewed and approved the study (No: 201703526). All animal experiments were carried out in accordance with the National Institutes of Health's Guidelines for the Care and Use of Laboratory Animals.

Open Access Statement: This is an Open Access article 


\section{Page 10 of 11}

distributed in accordance with the Creative Commons Attribution-NonCommercial-NoDerivs 4.0 International License (CC BY-NC-ND 4.0), which permits the noncommercial replication and distribution of the article with the strict proviso that no changes or edits are made and the original work is properly cited (including links to both the formal publication through the relevant DOI and the license). See: https://creativecommons.org/licenses/by-nc-nd/4.0/.

\section{References}

1. Foxman B. The epidemiology of urinary tract infection. Nat Rev Urol 2010;7:653-60.

2. Plotnikov EY, Morosanova MA, Pevzner IB, et al. Protective effect of mitochondria-targeted antioxidants in an acute bacterial infection. Proc Natl Acad Sci U S A 2013;110:E3100-8.

3. Vysakh A, Raji NR, Suma D, et al. Role of antioxidant defence, renal toxicity markers and inflammatory cascade in disease progression of acute pyelonephritis in experimental rat model. Microb Pathog 2017;109:189-94.

4. Pakkyara A, Jha A, Al Salmi I, et al. Gas in the kidney in asymptomatic Escherichia coli urinary tract infections in a patient with severe vesicoureteral reflex. Saudi J Kidney Dis Transpl 2019;30:706-9.

5. Lee SH, Choi T, Choi J, et al. Differences between Risk Factors for Sepsis and Septic Shock in Obstructive Urolithiasis. J Korean Med Sci 2020;35:e359.

6. Zhang YL, Feng XE, Chang RR, et al. Therapeutic effects of 5,2'-dibromo-2,4',5'-trihydroxydiphenylmethanone (LM49) in an experimental rat model of acute pyelonephritis by immunomodulation and antiinflammation. Int Immunopharmacol 2018;62:155-64.

7. Canning DA. Re: Obesity and a febrile urinary tract infection: dual burden for young children? J Urol 2015;193:990.

8. Mahyar A, Ayazi P, Gholmohammadi P, et al. The role of overweight and obesity in urinary tract infection in children. Infez Med 2016;24:38-42.

9. Mazaki-Tovi S, Romero R, Vaisbuch E, et al. Low circulating maternal adiponectin in patients with pyelonephritis: adiponectin at the crossroads of pregnancy and infection. J Perinat Med 2010;38:9-17.

10. Arita Y, Kihara S, Ouchi N, et al. Paradoxical decrease of an adipose-specific protein, adiponectin, in obesity. Biochem Biophys Res Commun 1999;257:79-83.

11. Bastard JP, Maachi M, Lagathu C, et al. Recent advances in the relationship between obesity, inflammation, and insulin resistance. Eur Cytokine Netw 2006;17:4-12.

12. Wang ZV, Scherer PE. Adiponectin, the past two decades. J Mol Cell Biol 2016;8:93-100.

13. Cheng MY, Liu H, Zhang TM, et al. Different forms of adiponectin reduce the apoptotic and damaging effect of cigarette smoke extract on human bronchial epithelial cells. Exp Ther Med 2016;12:4168-74.

14. Hao R, Su G, Sun X, et al. Adiponectin attenuates lipopolysaccharide-induced cell injury of H9c2 cells by regulating AMPK pathway. Acta Biochim Biophys Sin (Shanghai) 2019;51:168-77.

15. Wörmann SM, Song L, Ai J, et al. Loss of P53 Function Activates JAK2-STAT3 Signaling to Promote Pancreatic Tumor Growth, Stroma Modification, and Gemcitabine Resistance in Mice and Is Associated With Patient Survival. Gastroenterology 2016;151:180-193.e12.

16. Liu Z, Gan L, Zhou Z, et al. SOCS3 promotes inflammation and apoptosis via inhibiting JAK2/STAT3 signaling pathway in 3T3-L1 adipocyte. Immunobiology 2015;220:947-53.

17. Wang B, Guo H, Li X, et al. Adiponectin Attenuates Oxygen-Glucose Deprivation-Induced Mitochondrial Oxidative Injury and Apoptosis in Hippocampal HT22 Cells via the JAK2/STAT3 Pathway. Cell Transplant 2018;27:1731-43.

18. Svensson M, Yadav M, Holmqvist B, et al. Acute pyelonephritis and renal scarring are caused by dysfunctional innate immunity in $\mathrm{mCxcr} 2$ heterozygous mice. Kidney Int 2011;80:1064-72.

19. Arifin WN, Zahiruddin WM. Sample Size Calculation in Animal Studies Using Resource Equation Approach. Malays J Med Sci 2017;24:101-5.

20. Zhang $\mathrm{T}, \mathrm{Wu} \mathrm{KY}, \mathrm{Ma} \mathrm{N}$, et al. The $\mathrm{C} 5 \mathrm{a} / \mathrm{C} 5 \mathrm{aR} 2$ axis promotes renal inflammation and tissue damage. JCI Insight 2020;5:e134081.

21. Zhong X, He J, Zhang X, et al. UCP2 alleviates tubular epithelial cell apoptosis in lipopolysaccharide-induced acute kidney injury by decreasing ROS production. Biomed Pharmacother 2019;115:108914.

22. Valentino L, Pierre J. JAK/STAT signal transduction: regulators and implication in hematological malignancies. Biochem Pharmacol 2006;71:713-21.

23. Liu J, Wang J, Ning Y, et al. The inhibition of miR-101a-3p alleviates H/R injury in H9C2 cells by regulating the JAK2/STAT3 pathway. Mol Med Rep 2020;21:89-96.

24. Tu Z, Tan X, Li S, et al. The therapeutic effect of controlled reoxygenation on chronic hypoxia-associated 
brain injury. Biol Open 2019;8:bio039370.

25. Jiang X, Guo CX, Zeng XJ, et al. A soluble receptor for advanced glycation end-products inhibits myocardial apoptosis induced by ischemia/reperfusion via the JAK2/ STAT3 pathway. Apoptosis 2015;20:1033-47.

26. Yang $Y$, Duan $W$, Jin Z, et al. JAK2/STAT3 activation by melatonin attenuates the mitochondrial oxidative damage induced by myocardial ischemia/reperfusion injury. J Pineal Res 2013;55:275-86.

27. Li Y, Shi X, Li J, et al. Knockdown of KLF11 attenuates hypoxia/reoxygenation injury via JAK2/STAT3 signaling in H9c2. Apoptosis 2017;22:510-8.

28. Liu X, Zhang X, Zhang J, et al. Diosmin protects against cerebral ischemia/reperfusion injury through activating JAK2/STAT3 signal pathway in mice. Neuroscience 2014;268:318-27.

29. Kong F, Sun Y, Song W, et al. MiR-216a alleviates LPSinduced acute lung injury via regulating JAK2/STAT3 and NF- $\kappa$ B signaling. Hum Cell 2020;33:67-78.

30. Meares GP, Liu Y, Rajbhandari R, et al. PERK-dependent activation of JAK1 and STAT3 contributes to endoplasmic reticulum stress-induced inflammation. Mol Cell Biol 2014;34:3911-25.

31. Ma C, Wang Y, Dong L, et al. Anti-inflammatory effect of resveratrol through the suppression of NF- $\mathrm{BB}$ and JAK/ STAT signaling pathways. Acta Biochim Biophys Sin (Shanghai) 2015;47:207-13.

32. He $\mathrm{P}$, Zhang $\mathrm{D}, \mathrm{Li} \mathrm{H}$, et al. Hepatitis B virus $\mathrm{X}$ protein modulates apoptosis in human renal proximal tubular epithelial cells by activating the JAK2/STAT3 signaling pathway. Int J Mol Med 2013;31:1017-29.

33. Lu Y, Ma Y, Wang R, et al. Adiponectin inhibits vascular smooth muscle cell calcification induced by betaglycerophosphate through JAK2/STAT3 signaling pathway. J Biosci 2019;44:86.

34. Wang T, Mao X, Li H, et al. N-Acetylcysteine and allopurinol up-regulated the Jak/STAT3 and PI3K/Akt pathways via adiponectin and attenuated myocardial postischemic injury in diabetes. Free Radic Biol Med 2013;63:291-303.

Cite this article as: Dai G, He Y. Adiponectin alleviated renal cell apoptosis and inflammation via inactivation of JAK2/ STAT3 signal pathway in an acute pyelonephritis mouse model. Ann Transl Med 2021;9(16):1311. doi: 10.21037/atm-21-3325
35. Vansaun MN. Molecular pathways: adiponectin and leptin signaling in cancer. Clin Cancer Res 2013;19:1926-32.

36. Zhang $M$, Wang X, Wang X, et al. Oxymatrine protects against myocardial injury via inhibition of JAK2/STAT3 signaling in rat septic shock. Mol Med Rep 2013;7:1293-9.

37. Zhou Y, Xu W, Zhu H. CXCL8(3-72) K11R/G31P protects against sepsis-induced acute kidney injury via NF$\kappa \mathrm{B}$ and JAK2/STAT3 pathway. Biol Res 2019;52:29.

38. Tanaka T, Narazaki M, Kishimoto T. IL-6 in inflammation, immunity, and disease. Cold Spring Harb Perspect Biol 2014;6:a016295.

39. Nanda N, Juthani-Mehta M. Novel biomarkers for the diagnosis of urinary tract infection-a systematic review. Biomark Insights 2009;4:111-21.

40. Azab S, Zakaria M, Raafat M, et al. The combination of urinary IL - 6 and renal biometry as useful diagnostic tools to differentiate acute pyelonephritis from lower urinary tract infection. Int Braz J Urol 2016;42:810-6.

41. Sheu JN, Chen MC, Cheng SL, et al. Urine interleukin1beta in children with acute pyelonephritis and renal scarring. Nephrology (Carlton) 2007;12:487-93.

42. Idriss HT, Naismith JH. TNF alpha and the TNF receptor superfamily: structure-function relationship(s). Microsc Res Tech 2000;50:184-95.

43. Mohkam M, Asgarian F, Fahimzad A, et al. Diagnostic potential of urinary tumor necrosis factor-alpha in children with acute pyelonephritis. Iran J Kidney Dis 2009;3:89-92.

44. Mohkam M. Novel Urinary Biomarkers for Diagnosis of Acute Pyelonephritis in Children. Iran J Kidney Dis 2020;14:1-7.

45. Safioleas K, Giamarellos-Bourboulis EJ, Carrer DP, et al. Reverse kinetics of angiopoietin-2 and endotoxins in acute pyelonephritis: Implications for anti-inflammatory treatment? Cytokine 2016;81:28-34.

46. Docheva N, Romero R, Chaemsaithong P, et al. The profiles of soluble adhesion molecules in the "great obstetrical syndromes". J Matern Fetal Neonatal Med 2019;32:2113-36.

(English Language Editors: B. Meiser and J. Gray) 